\title{
Transient Respiratory Response to Hypercapnia: Analysis via a Cardiopulmonary Simulation Model
}

\author{
A. Albanese, Student Member, IEEE, N. W. Chbat, Member, IEEE, M. Ursino
}

\begin{abstract}
In recent years, our group has developed a comprehensive cardiopulmonary (CP) model that comprises the heart, systemic and pulmonary circulations, lung mechanics and gas exchange, tissue metabolism, and cardiovascular and respiratory control mechanisms. In this paper, we analyze the response of the model to hypercapnic conditions and hence focus on the chemoreflex control mechanism. Particularly, we have enhanced the peripheral chemoreceptor model in order to better reflect respiratory control physiology. Using the $\mathrm{CO}_{2}$ fraction in the inspired air as input to the $\mathrm{CP}$ model, we were able to analyze the transient response of the system to $\mathrm{CO}_{2}$ step input at different levels, in terms of alveolar gas partial pressures, tidal volume, minute ventilation and respiratory frequency. Model predictions were tested against experimental data from human subjects [1]. Results show good agreement for all the variables under study during the transient phases and low root mean square errors at steady state. This indicates the potential for the model to be used as a valid tool for clinical practice and medical research, providing a complementary way to experience-based clinical decisions.
\end{abstract}

\section{INTRODUCTION}

$\mathrm{S}_{\mathrm{c}}$ IMULATIONS of hypercapnia by means of a comprehensive cardiopulmonary model offer useful information from physiological and clinical perspectives. First, the complex interactions between the cardiovascular and respiratory systems can be analyzed and understood; the individual components of the physiological control mechanisms of cardio-pulmonary regulation can be evaluated; and, the overall response of the system can be studied in a more rigorous and quantitative manner. Further, hypercapnic respiratory failure (usually hypoventilation-induced) is very commonly observed in intensive care unit (ICU) patients suffering, for instance, from chronic obstructive pulmonary disease (COPD), sleep apnea, obesity hypoventilation syndrome, and others. Having a model that is able to emulate the patient and predict the response to different levels of inspired gas fractions could be a valuable tool to provide clinical decision supportive information in the ICU.

\section{METHODS}

The cardiopulmonary model includes description of the cardiovascular system, lung mechanics, gas exchange in the alveoli and tissue metabolism along with the main control mechanisms involved in cardiopulmonary regulation.

Manuscript received April 15, 2011.

A. Albanese is with the Department of Biomedical Engineering, Columbia University, New York, NY 10027 (e-mail: aa2932@columbia.edu).

N. W. Chbat is with Philips Research North America. Briarcliff Manor, NY 10510; Corresponding author phone: 914-945-6238; e-mail: chbat@philips.com.

M. Ursino is with the Department of Electronics, Computer Science and Systems, University of Bologna, Bologna, 40136 Italy (e-mail: mauro.ursino@unibo.it).
Particularly, cardiovascular control is mediated by baroreceptors, lung stretch receptors, peripheral chemorecptors, local autoregulation and a direct effect of the central nervous system (CNS ischemic response), whereas respiratory control is mediated by central and peripheral chemorecptors. Description of the model has been provided in [2]. For this reason, only a quick overview of the model is described next, with an emphasis on the new aspects of the chemoreflex mechanism and related hypercapnic simulations.

\section{A. Cardiovascular Model}

The cardiovascular model has been adapted from previous work [3], [4]. It includes pulsatile description of the right and left hearts, as well as systemic and pulmonary circulations. Cardiovascular function is assumed under the control of the autonomic nervous system and local metabolic mechanisms (autoregulation). Details on the model can be found in [3], [4].

\section{B. Lung Mechanics and Gas Exchange Models}

The lung mechanics dynamic model has been adapted from an existing model [5]. It includes 4 compartments: larynx, trachea, bronchea and alveoli. As shown in Fig. 1, each compartment is represented as combination of a linear compliance and a linear resistance. The model can be driven by two different pressure source generators: a thoracic source $U_{t}$, which mimics the action of the respiratory muscles, and an external source $U_{m}$, which mimics the effect of a mechanical ventilator. The two sources can be separately switched on to simulate either spontaneous breathing or artificial ventilation, but they can also act at the same time to simulate simultaneous natural and artificial breathing condition (assisted ventilation).

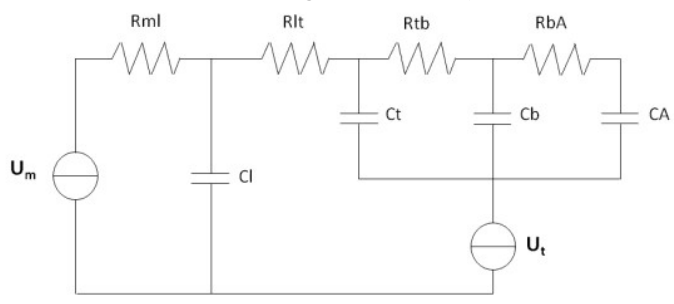

Fig. 1. Electrical analog of the lung mechanics system. C:capacitance, R:resistance. Subscripts: m:mouth, 1:larynx, t:trachea, b:bronchi, A:alveoli

The thoracic pressure generator $U_{t}$ is modeled as a sinusoidal function of amplitude $A_{t}$ and frequency $f_{t}$, with a negative bias term to account for longer exhalation time:

$U_{t}=A t \cdot \sin (2 \cdot \pi \cdot f t \cdot t)-B$

The gas exchange model includes dead space, alveoli and pulmonary capillaries. Equations governing the model are derived based on conservation of mass using gas fractions in the inspired air as inputs. Oxygen and carbon dioxide metabolic processes at the tissue level are also described by way of differential equations based on mass conservation, assuming 
that $\mathrm{O}_{2}$ and $\mathrm{CO}_{2}$ metabolic rates are constant and known. Finally, pulmonary shunts, transport circulatory delays and empirical dissociation curves [6] are also included in the model.

\section{Respiratory Control Model}

The automatic control of breathing in humans involves central and peripheral chemoreceptors that increase pulmonary ventilation when stimulated. Inputs to the chemoreflex are the cellular hydrogen ion concentrations at both central and peripheral sites, often expressed in terms of partial pressure of oxygen and carbon dioxide in the blood. Particularly, peripheral chemoreceptors are sensitive to both $\mathrm{Pa}_{\mathrm{O} 2}$ and $\mathrm{Pa}_{\mathrm{CO} 2}$, whereas central chemoreceptors are sensitive to $\mathrm{Pa}_{\mathrm{CO} 2}$ only. In addition, respiration is driven by an intrinsic respiratory rhythm generator, which produces basal ventilation when chemoreceptors are not stimulated [7].

The majority of the ventilation control models available in literature assume that chemoreceptors act on the respiratory system either by directly changing minute ventilation, $\dot{V}$, or by modifying tidal volume $\mathrm{V}_{\mathrm{T}}$ and respiratory frequency $f$, and ultimately affecting minute ventilation. In these models, a set of static or dynamic equations coupling $\mathrm{Pa}_{\mathrm{O} 2}$ and $\mathrm{Pa}_{\mathrm{CO} 2}$ (or some surrogates of these variables) to $\dot{\mathrm{V}}$ (or $\mathrm{V}_{\mathrm{T}}$ and $f$ ) is used to describe the whole respiration control system. Hence, the physiological link between the controller (i.e. the receptors and the respiratory centers in the brain) and the actuator (i.e. the respiratory muscles) and the description of lung dynamics are missing in these models. The only available model incorporating this aspect that we are aware of is the one reported in [8], [9]. This model, however, is not suitable for integration into our cardiopulmonary model since the input quantity to the central chemoreceptors is hydrogen ion concentration in the cerebrospinal fluid, which is not a variable in our model.

Hence, we propose a new ventilation control model where chemoreceptors act on respiration by modifying the amplitude $A_{t}$ and the frequency $f_{t}$ of the sinusoidal pressure generator $U_{t}$ (1). Since there is no evidence in the literature of active interaction between the two distinct central and peripheral chemoreceptor mechanisms, a simple additive relationship between central and peripheral responses has been assumed. Contributions from the chemoreceptors are then added to the basal values from a constant respiratory rhythm generator to determine the total respiratory drive (see Fig. 2).

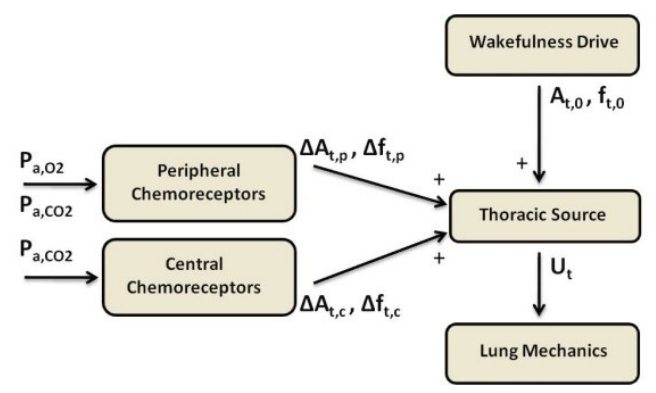

Fig. 2. Schematic block diagram of the respiratory control model. $A_{t, 0}$ and $\mathrm{f}_{\mathrm{t}, 0}$ are the basal values of amplitude and frequency, whereas $\Delta \mathrm{A}_{\mathrm{t}, \mathrm{c}}, \Delta \mathrm{f}_{\mathrm{t}, \mathrm{c}}$ and $\Delta \mathrm{A}_{\mathrm{t}, \mathrm{p}}, \Delta \mathrm{f}_{\mathrm{t}, \mathrm{p}}$ represent the changes in amplitude and frequency induced by central and peripheral chemoreceptor activations, respectively.
Following the approach in [4], the central chemoreceptor mechanism is described as a first-order dynamic system with a pure delay, having as input the variation of $\mathrm{P}_{\mathrm{CO} 2}$ in the arterial blood (assuming that variations of $\mathrm{P}_{\mathrm{CO} 2}$ in arterial blood and in the medulla are proportional). Unlike what is assumed in [4], outputs of the dynamic system are here the changes in amplitude and frequency of the thoracic source:

$$
\begin{aligned}
& \frac{d \Delta A t, c}{d t}=\frac{1}{\tau c, A}\left\{-\Delta A t, c+g_{c, A}\left[P a, C O 2\left(t-D_{c}\right)-P a C O 2, n\right]\right\} \\
& \frac{d \Delta f t, c}{d t}=\frac{1}{\tau c, f}\left\{-\Delta f t, c+g_{c, f}[P a, C O 2(t-D c)-P a C O 2, n]\right\}
\end{aligned}
$$

where, $\mathrm{P}_{\mathrm{aCO} 2, \mathrm{n}}$ is the normal value of $\mathrm{P}_{\mathrm{a}, \mathrm{CO} 2}(40 \mathrm{mmHg}), \mathrm{g}_{\mathrm{c}, \mathrm{A}}$ and $g_{c, f}$ are gain factors for the regulatory mechanisms of $A_{t}$ and $f_{t}$, respectively, $\mathrm{D}_{\mathrm{c}}$ is the pure delay and finally $\tau_{\mathrm{c}, \mathrm{A}}$ and $\tau_{\mathrm{c}, \mathrm{f}}$ are time constants of the two mechanisms, respectively.

The description of the peripheral chemoreceptors has been modified compared to our previous version of the model in [2]. Based on the work in [4], peripheral chemoreflex is described as a two-stage transduction mechanism: $\mathrm{Pa}_{\mathrm{O} 2}$ and $\mathrm{Pa}_{\mathrm{CO} 2}$ variations are first transduced into electrical activity of the peripheral chemoreceptor fibers, which are then converted into variations of amplitude and frequency of the thoracic source generator. As suggested in [4], relationships analogous to those in (2) and (3) have been used to describe the second stage:

$$
\begin{aligned}
& \frac{d \Delta A t, p}{d t}=\frac{1}{\tau p, A}\left\{-\Delta A t, p+g p, A\left[f a p d\left(t-D_{p}\right)-f_{a p c, n}\right]\right\} \\
& \frac{d \Delta f t, p}{d t}=\frac{1}{\tau p, f}\left\{-\Delta f t, p+g p, f\left[f_{a p} d\left(t-D_{p}\right)-f_{a p c, n}\right]\right\}
\end{aligned}
$$

where $f_{a p c, n}$ is the basal value of the peripheral chemoreceptor activity (i.e. the value computed during normoxia and normocapnia), and convention for gains, delay and time constants is equivalent to the one adopted in (2) and (3). Conversely, the relation describing the first transduction stage has been modified from that proposed in [4]. This was necessary since the equations proposed in [4] were not able to reproduce the overshoot and undershoot characterizing the typical peripheral chemoreceptors activity patterns, shown in Fig. 3, observed in humans in response to a $\mathrm{CO}_{2}$ step input [10]. Two different models for afferent peripheral chemorecptors activity, reported in [8] and [11], were considered in order to replace the equations in [4]. Results proved that only the model in [11] was able to reproduce the overshoot and undershoot experimentally observed in humans, and for this reason we incorporated this model into our peripheral chemoreflex description. The block diagram of the model in [11] is shown in Fig. 4.

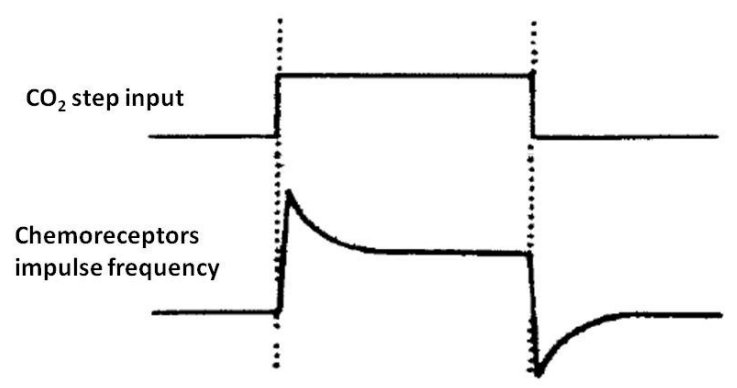

Fig. 3. Diagrams of time-dependent single-fiber responses of perfused carotid chemoreceptors to up and down steps of $\mathrm{CO}_{2}$. Adapted from [10]. 


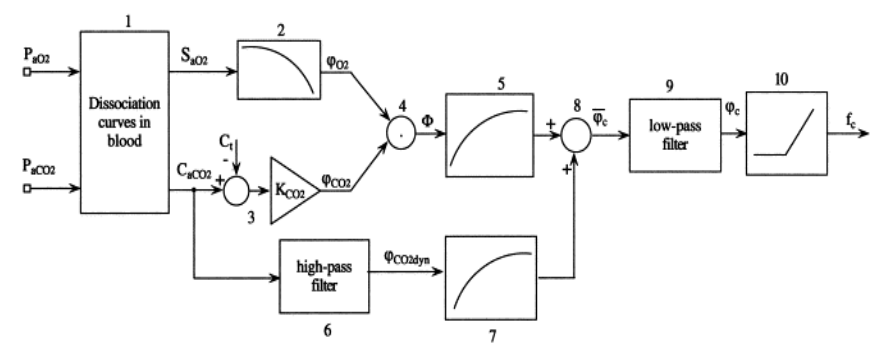

Fig. 4. Block diagram describing the peripheral chemoreceptor model (adapted from [11]). Inputs to the model are $\mathrm{O}_{2}$ saturation and $\mathrm{CO}_{2}$ concentration. Output of the model is the peripheral chemorecptors activity $\mathrm{f}_{\mathrm{c}}$. Details on individual blocks can be found in [11].

\section{RESULTS AND DISCUSSION}

In order to illustrate the possibilities offered by the model, hypercapnic conditions were simulated and compared to published human data. Simulations were performed with the cardiopulmonary model in closed-loop configuration and using the inspired gas concentrations $\mathrm{F}_{\mathrm{ICO} 2}$ and $\mathrm{F}_{\mathrm{IO} 2}$ as input. A step input from $0 \%$ to $7 \%$ was used for $\mathrm{F}_{\mathrm{ICO} 2}$, whereas $\mathrm{F}_{\mathrm{IO} 2}$ was kept fixed to its normal room ambient value of $21 \%$. The gains and time constants of the peripheral and central chemoreceptors where chosen to match the experimental results reported in [1] while adhering to physiological constraints. The central and peripheral delays were given the values reported in [4]. Based on the data in [1], time constants for the breathing frequency response were given higher values than the time constants for the amplitude response. These time constants for both the central and peripheral mechanisms were assumed to have different values during the up and down-transient phases.

Simulation results are shown in Fig. 5 and are compared to the experimental data reported in [1]. As we can see, the model agrees quite well with the experimental results both in the steady-state and transient phases for all the variables under study. The most significant level of discrepancy between model predictions and experimental data is represented by the overshoot in $\mathrm{P}_{\mathrm{ACO} 2}$, which was not observed in the experimental data, and by the much more pronounced undershoot in the simulated $\mathrm{P}_{\mathrm{ACO} 2}$. This discrepancy, however, could be justified by taking into account the averaging of the experimental data, since the author of [1] reported that averaging of data over a number of subjects tended to smear the undershoot in $\mathrm{P}_{\mathrm{ACO} 2}$ and that natural oscillations in partial pressures were observed in individual subjects. Moreover, the presence of overshoot and undershoot in the predicted $\mathrm{P}_{\mathrm{ACO} 2}$ waveform in response to the same kind of $\mathrm{CO}_{2}$ stimulus has been observed in recently proposed models ([12], [13]). Further, we could hypothesize that the model-predicted overshoot and undershoot responses can be ascribed to the absence of a $\mathrm{CO}_{2}$ buffering system, otherwise physiologically present in human subjects, which would have prevented such drastic changes in $\mathrm{P}_{\mathrm{ACO} 2}$.

Fig. 6 shows the separate contributions of central and peripheral chemoreceptors to the total thoracic source frequency and amplitude. As we see, the central chemoreceptors seem to play the most important role in triggering the respiratory response to hypercapnia. In order to explain this interesting phenomenon let us analyze the peripheral chemoreceptors firing frequency time pattern. From Fig. 7, at the $\mathrm{CO}_{2}$ stimulus onset time, the peripheral chemoreceptor activity initially increases rapidly beyond its normal resting value. Beyond this time, these receptors are essentially silent during the remaining 7\% $\mathrm{CO}_{2}$ breathing period. The reason for this behavior is essentially due to the multiplicative effect of oxygen and carbon dioxide at the peripheral chemoreceptors site. Basically, right after the $\mathrm{CO}_{2}$ stimulus the peripheral chemoreceptors are highly stimulated by the increased $\mathrm{CO}_{2}$ level in the arterial blood. This stimulating effect of hypercapnia on peripheral chemoreceptors, however, is soon counteracted by the progressively increasing $\mathrm{O}_{2}$ level (hyperoxia) that is in turn due to the increased minute ventilation. As a result, the firing frequency of the peripheral chemoreceptors is rapidly reset to values close to those in a resting state where they have essentially no effect on triggering the respiratory response.
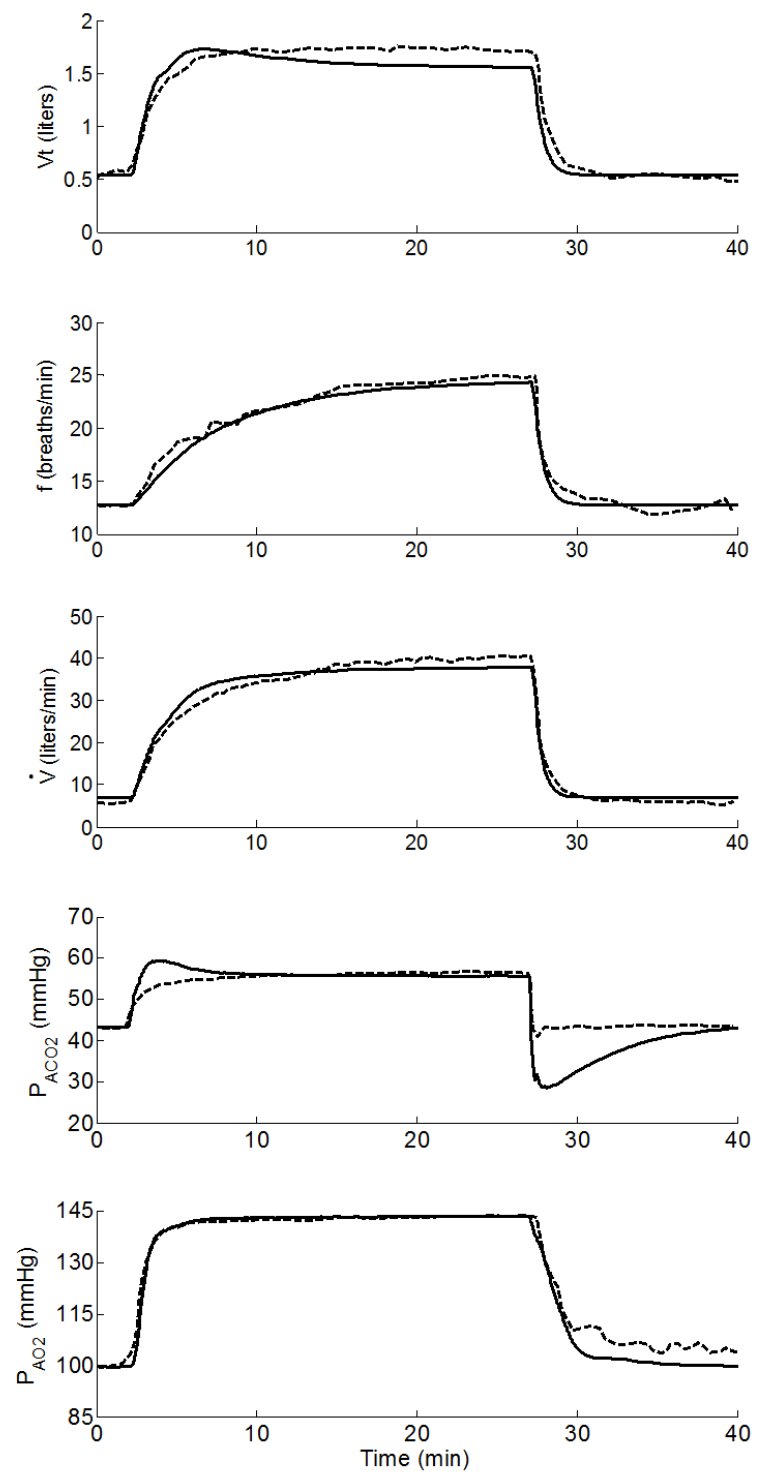

Fig. 5. Respiratory response to a 7\% $\mathrm{CO} 2$ step input performed at $2 \mathrm{~min}$ and lasting $25 \mathrm{~min}$. Continuous lines are model results; dashed lines are experimental data redrawn from [1]. Experimental data represents the average over 15 subjects. Time continuous model outputs were averaged over $10 \mathrm{sec}$ in order to follow the same signal processing procedure used in [1].

In order to check the agreement between model prediction and experimental human data at different level of hypercapnic stimuli, simulations were also performed at 3\%,5\% and $6 \%$ $\mathrm{CO}_{2}$ step input levels. Fig. 8 compares the steady-state changes of the main respiratory variables with the data reported in [1]. 

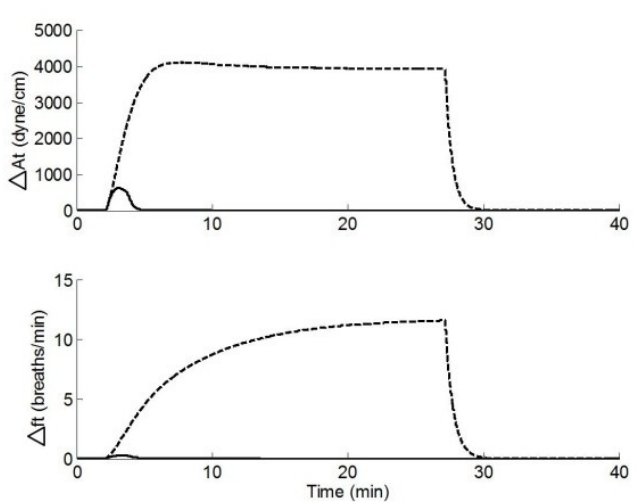

Fig. 6. Separate contributions of central (dashed lines) and peripheral chemoreceptors (continuous lines) to $\Delta \mathrm{A}_{\mathrm{t}}$ and $\Delta \mathrm{f}_{\mathrm{t}}$, the total changes in frequency and amplitude of the thoracic source.

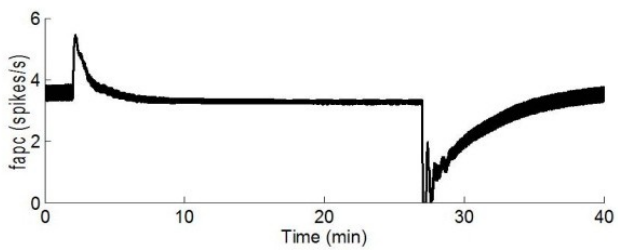

Fig. 7. Peripheral chemoreceptors activity in response to the $7 \% \mathrm{CO}_{2}$ step input as computed by the model.
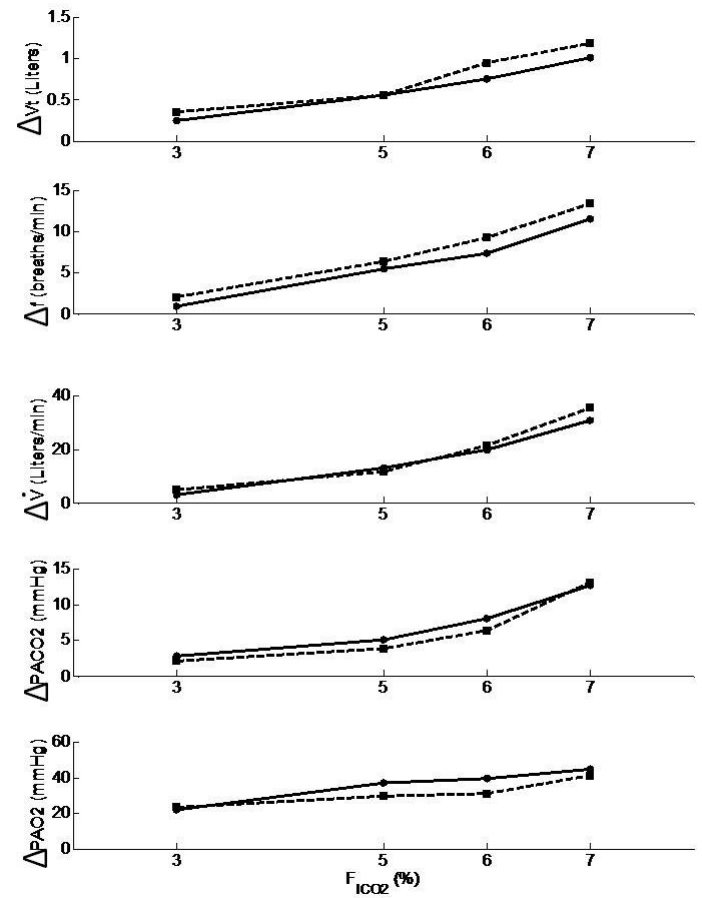

Fig. 8. Steady-state changes for tidal volume, respiratory rate, minute ventilation, and alveolar gas partial pressures in response to $\mathrm{CO}_{2}$ step input at different levels. Continuous line: model results; dashed line: experimental data from [1].

It shows the correct trending of the respiratory response in terms of minute ventilation, tidal volume, respiratory rate and alveolar gas partial pressures. The root mean square errors (RMSE) in the predicted steady-state changes for each variable under study were also computed across the $\mathrm{F}_{\mathrm{ICO} 2}$ range. The RMSE is: $5.9 \mathrm{mmHg}$ for $\mathrm{P}_{\mathrm{AO} 2} ; 1.1 \mathrm{mmHg}$ for $\mathrm{P}_{\mathrm{ACO} 2} ; 2.7 \mathrm{~L} / \mathrm{min}$ for minute ventilation; $1.5 \mathrm{breath} / \mathrm{min}$ for respiratory frequency and $0.1 \mathrm{~L}$ for tidal volume.

\section{CONCLUSION}

Encouraging results are obtained from our comprehensive cardiopulmonary model with normal parameter values, and simulation results are compared to experimental human data. The predicted respiratory response to different levels of hypercapnic stimuli agrees quite well with real human data for all the variables under study and in both steady-state and transient conditions. The root mean squared errors in the predicted steady-state changes are: $5.9 \mathrm{mmHg}$ for $\mathrm{P}_{\mathrm{AO} 2} ; 1.1$ $\mathrm{mmHg}$ for $\mathrm{P}_{\mathrm{ACO} 2} ; 2.7 \mathrm{~L} / \mathrm{min}$ for minute ventilation; 1.5 breath/min for respiratory frequency and $0.1 \mathrm{~L}$ for tidal volume. Our model has feedback regulatory mechanisms for chemoreflex, lung stretch, baroreflex, autoregulation and CNS ischemic response. These regulate a cardiovascular system linked to lung mechanics, gas exchange and metabolic systems. Simulating hypercapnic respiratory failure could help in understanding COPD, sleep apnea, obesity hypoventilation syndrome and other diseases that are prevalent in the ICU. Moreover, by altering different parameter values, the model can be used to simulate other pathological conditions of clinical relevance, such as cardiogenic and hypovolumic shock, respiratory obstructions, hypoxia and apnea. Hence, the model could be used as a valuable tool to examine cardiopulmonary and metabolic changes in ICU patients and run different what if scenarios, thus providing useful information for clinical decision making.

\section{REFERENCES}

[1] W. J. Reynolds and H. T. Milhorn, Transient ventilatory response to graded hypercapnia in man. $J$ Appl Physiol 33: 47-54, 1972.

[2] N. W. Chbat, M. Giannessi, A. Albanese and M. Ursino, A comprehensive cardiopulmonary simulation model for the analysis of hypercapnic respiratory failure. Conf Proc IEEE Eng Med Biol Soc. 2009:5474-7, 2009.

[3] M. Ursino and E. Magosso, Acute cardiovascular response to isocapnic hypoxia. I. A mathematical model. Am J Physiol Heart Circ Physiol 279: H149-H165, 2000.

[4] E. Magosso and M. Ursino, A mathematical model of $\mathrm{CO} 2$ effect on cardiovascular regulation. Am J Physiol Heart Circ Physiol 281: H2036-H2052, 2001.

[5] V. Rideout, Respiratory System Modelling. in Mathematical and Computer Modelling of Physiological Systems, Englewood Cliffs, New Jersey: Prentice-Hall, 1991, pp. 137-143.

[6] J. L. Spencer, E. Firouztale and R. B. Mellins, Computational expressions for blood oxygen and carbon dioxide concentrations. Ann Biomed Eng 7: 59-66, 1979.

[7] B. R. Fink, Influence of cerebral activity in wakefulness on regulation of breathing. J Appl Physiol 16: 15-20, 1961.

[8] K. Lu, J. W. Clark, Jr., F. H. Ghorbel, D. L. Ware, J. B Zwischenberger and A. Bidani, A whole body closed-loop model of gas exchange in human. Cardiovasc Eng 3: 1-19, 2003.

[9] K. Lu, J. W. Clark, Jr., F. H. Ghorbel, C. S. Robertson, D. L. Ware, J. B. Zwischenberger and A. Bidani, Cerebral autoregulation and gas exchange studied using a human cardiopulmonary model. $J$ Appl Physiol 84:1447-1469, 1998.

[10] D. J. C. Cunningham, Studies on arterial chemoreceptors in man. $J$ Physiol 384: 1-26, 1987.

[11] M. Ursino and E. Magosso, A theoretical analysis of the carotid body chemoreceptor response to $\mathrm{O} 2$ and $\mathrm{CO} 2$ pressure changes. Resp Physiol \& Neurob 130: 99-110, 2002.

[12] M. B. Wolf and R. P. Garner, A mathematical model of human respiration at altitude. Ann Biomed Eng 35(11): 2003-2022, 2007.

[13] S. Sokhanvar, J. Dargahi, M. Packirisamy and E. Esmailzadeh, Modeling of chemical control of human respiratory system. Biomed Mater Eng 15(6): 467-481, 2005. 